\title{
Fabrication and Characterization of Percolating Nanocluster Devices
}

\author{
Ayesh $\mathbf{A l}^{*}$, Mahmoud ST, Qamhieh N and Karam ZA \\ United Arab Emirates University, P.O. Box 17551, Al Ain, United Arab Emirates
}

\begin{abstract}
We are reporting on the fabrication and characterization of palladium (Pd) nanocluster devices. Pd nanoclusters were synthesized inside a dc magnetron sputtering chamber, where the plasma vaporizes the target's material and nanoclusters are formed by inert gas condensation. The size distribution of the produced nanoclusters is investigated using quadrupole mass filter.

To fabricate a device, nanoclusters are self-assembled on a $\mathrm{SiO}_{2} / \mathrm{Si}$ substrate that contains a pair of pre-formed electrical electrodes defined by optical lithography. The electric current between the electrodes is monitored during nanoclusters deposition. A sharp increase in the current indicates the formation of a continuous network of clusters, thus, nanocluster deposition is abruptly stopped. Electronic transport of the device is investigated by systematic currentvoltage measurements as a function of temperature.
\end{abstract}

Keywords: Pd nanoclusters; Inert-gas condensation; Sputtering; Nanocluster devices

\section{Introduction}

Investigating nanocluster properties is of strategic importance, since the properties of nanoclusters can be tuned by controlling their size which creates innovative materials and devices. Recently, research has been focused on palladium (Pd) nanoclusters because of possible applications in the fields of hydrogen storage [1,2], hydrogen detection [3], and catalysts [4,5]. Therefore, intensive research is required to develop nanocluster sources to produce more intensive nanocluster beam, and to provide further control of nanocluster size distributions $[6,7]$. Sputtering and inert gas-condensation is a standard candidate that combines both plasma sputtering and inert gas condensation without the complications of target heating [8]. In addition, plasma gas condensation is a predominant technology that can be used for large scale production by the industries to produce high quality metallic nanoclusters $[9,10]$. Nevertheless, only few systematic investigations were devoted to improve this technique to reach an optimal large scale production of nanoclusters $[8,10,11]$.

The growth of nanoclusters may involve either or both of the following processes: i) embryo formation via three-body collision (two sputtered atoms and an inert gas atom to remove their excess kinetic energy); and ii) nucleation of large nanoclusters by two-body collision through nanocluster-nanocluster collision and accepting atoms that have arrived to the nanocluster surface (atomic condensation) [12,13]. Hence, number of produced nanoclusters and nanoclusters' size are determined by the probability of the two-body and/or three-body collisions which are mainly dependent on the time nanoclusters spend within the growth region.

Nanoclusters deposited for a device fabrication are arranged randomly to form a network between electrical electrodes. The electrical conduction between each pair of nanoclusters is determined by the nature of connection at the interface between nanoclusters. Rigorous research effort is needed to address the electronic transport in Pd nanocluster devices.

In this work, the production mechanisms of Pd nanoclusters are investigated. In addition, the produced nanoclusters were deposited between electrical electrodes to study the charge carrier transport within the device.

\section{Experimental}

DC discharge magnetron sputtering technique was used to fabricate Pd nanoclusters inside an Ultra-High Vacuum compatible system (UHV) shown in Figure 1(a) [12]. UHV system consists of: i) $\mathrm{dc}$ magnetron sputtering source, ii) Quadrupole Mass Filter (QMF), and iii) deposition chamber. The plasma is ignited in a mixture of Ar and He inert gases by applying a dc voltage to create a dense vapor of atomic ions and small nanoclusters from the target. The nanoclusters are formed from the vapor by inert gas condensation within an aggregation region of length $\mathrm{L}$. The aggregation length can be varied during the experiment to optimize nanocluster production. The size of the produced nanoclusters depends on the aggregation length, the type of inert gases, their flow rates, and the sputtering discharge power (P) [8]. The nanocluster size was determined using a Quadrupole Mass Filter (QMF) [12]. To verify these sizes, Pd nanoclusters were

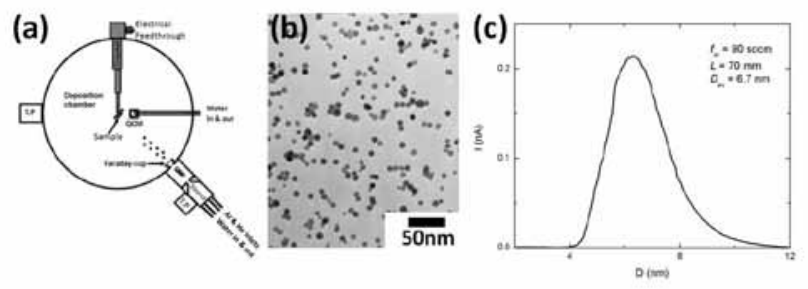

Figure 1: a) Schematic diagram of the ultra-high vacuum compatible system and the nanocluster source. b) TEM image of the Pd nanoclusters c) Nanocluster size distribution measured using the QMF. The nanoclusters were produced using $\mathrm{f}_{\mathrm{Ar}}=90 \mathrm{sccm}, \mathrm{f}_{\mathrm{He}}=0 \mathrm{sccm}, 7=90 \mathrm{~mm}$, and $\mathrm{P}=85 \mathrm{~W}$.

*Corresponding author: Ayesh Al, Department of Physics, United Arab Emirates University, P.O. Box 17551, Al Ain, United Arab Emirates, Tel: +971-3-7136315; E-mail: ayesh@uaeu.ac.ae

Received March 29, 2012; Accepted June 16, 2012; Published June 21, 2012

Citation: Ayesh Al, Mahmoud ST, Qamhieh N, Karam ZA (2012) Fabrication and Characterization of Percolating Nanocluster Devices. J Nanomed Nanotechol 3:143. doi:10.4172/2157-7439.1000143

Copyright: @ 2012 Ayesh Al, et al. This is an open-access article distributed under the terms of the Creative Commons Attribution License, which permits unrestricted use, distribution, and reproduction in any medium, provided the original author and source are credited. 
deposited on carbon-coated copper microgrids to be used for Philips CM10 Transmission Electron Microscope (TEM) imaging.

Nanoclusters were deposited on a $\mathrm{SiO}_{2} / \mathrm{Si}$ substrate with preformed $\mathrm{Au} / \mathrm{NiCr}$ contacts, which are $5 \mu \mathrm{m}$ apart, defined by standard optical lithography. The substrate was mounted on a cryostat finger inside the deposition chamber. The electrical conductivity of the sample was observed during nanoclusters deposition process. Subsequently, when nanoclusters form at least one continuous network between the electrodes, a sharp rise in the conductivity was observed. The nanoclusters deposition was abruptly stopped using an automatic gate valve. Electrical measurements as a function of temperature were conducted on the device using a Keithley 238 source measuring unit.

\section{Results and Discussion}

Pd nanoclusters were produced using an argon gas flow rare $\left(\mathrm{f}_{\mathrm{Ar}}\right)$ of $90 \mathrm{sccm}, \mathrm{P}=85 \mathrm{~W}$, and $\mathrm{L}=70 \mathrm{~mm}$. The TEM image and size distribution (as measured using the QMF) of the produced Pd nanoclusters are shown in Figure $1 \mathrm{~b}$ and Figure 1c. They are in good agreement, and reveal an average size of $\sim 6.7 \mathrm{~nm}$.

To investigate the controllability of the Pd nanocluster size, Helium $(\mathrm{He})$ gas was introduced into the chamber while maintaining $\mathrm{f}_{\mathrm{Ar}}$ at $40 \mathrm{sccm}, \mathrm{L}$ at $60 \mathrm{~mm}$, and $\mathrm{P}$ at $20.4 \mathrm{~W}$. The dependence of the mean nanocluster size on $\mathrm{f}_{\mathrm{He}}$ is shown in Figure 2. The figure shows that the mean nanocluster diameter decreases as $\mathrm{f}_{\mathrm{He}}$ increases. This drop in the diameter of nanoclusters at high Helium flow rate could be attributed to the fact that $\mathrm{He}$ is more efficient in sweeping the clusters out of the condensation region compared to Argon (Ar).

Cooling energetic metallic atoms sputtered from the target by Helium gas leads to the nucleation of nanoclusters. The nucleation of small nanocluster "seed" is followed by the growth of the seeds into large nanoclusters [14]. Their growth depends upon panoply of interatomic collisions such as three-body and two-body. In a three-body collision, a cold Helium (He) atom removes excess kinetic energy from the sputtered metallic atoms. Three-body collision occurs more frequently in a highly dense vapor than in normal gas condition, requiring a high sputter rate, and thus, high inert gas pressure. Therefore, the drop in the nanocluster size with inert gas flow rate observed in Figure 2 can be assigned to the dominance of the three-body collision mechanism $[11,1215,16]$.

Moreover, the nanocluster size depends strongly on the collision probability, hence, on the nucleation time (defined as the time spent by the nanocluster within the growth region). Therefore, the decrease of the mean diameter with Helium flow rate could be attributed to the decrease in the nucleation time. Herein, the viscosity of Helium gas is less than that of Argon, so that Helium gas stream is extracted out of the nucleation chamber more effectively than Argon [17]. The increase in Helium flow rate results in a high drift velocity of nanoclusters, consequently the nucleation time decreases. Thus, increasing Helium gas flow rate reduces the nucleation time and produces a smaller nanocluster.

The dependence of the mean nanocluster diameter on the aggregation length for $\mathrm{f}_{\mathrm{Ar}}=40 \mathrm{sccm}, \mathrm{f}_{\mathrm{He}}=15 \mathrm{sccm}, \mathrm{P}=20.4 \mathrm{~W}$ is shown in Figure 3. The figure shows that the mean diameter increases with $\mathrm{L}$ as a result of the increase in the nucleation time. Nanoclusters grow further for longer $L$ because they remain longer within the nucleation region. It is interesting to notice that small nanoclusters with diameter of about $2 \mathrm{~nm}$ are produced.
The area under a size distribution curve (for example Figure 1(c)) gives an indication of the number of measured nanoclusters. Figure 2 and Figure 3 show the dependence of the area under each size distribution curve on $\mathrm{f}_{\mathrm{He}}$ (Figure 2) and $\mathrm{L}$ (Figure 3). The figures show that the number of measured nanoclusters decreases with increasing $\mathrm{f}_{\mathrm{He}}$, while it increases with $\mathrm{L}$.

Mixing Argon with Helium gas is expected to cause a partial consumption of electrical power to ionize $\mathrm{He}$ gas whose sputtering yield is one order of magnitude smaller than that of Argon gas $[8,17]$. Therefore, increasing Helium flow rate would reduce the density of the sputtered material and consequently the number of produced nanoclusters. Increasing the aggregation length increases the nucleation time and hence increases the probability of the three-body collision [18]. Consequently, increases the size as well as the number of nanoclusters' seeds.

Figure 4(a) shows a Scanning Electron Microscopy (SEM) image of nanoclusters as deposited between the electrical electrodes. The nanoclusrters used for the device fabrication were produced using the same conditions as in Figure 1(c). The current-voltage (I(V)) measurements at room temperature and $42 \mathrm{~K}$ are shown in Figure 4(b). The figure reveals a transition from linear to non-linear characteristics as the temperature decreases. This transition is expected if electronic

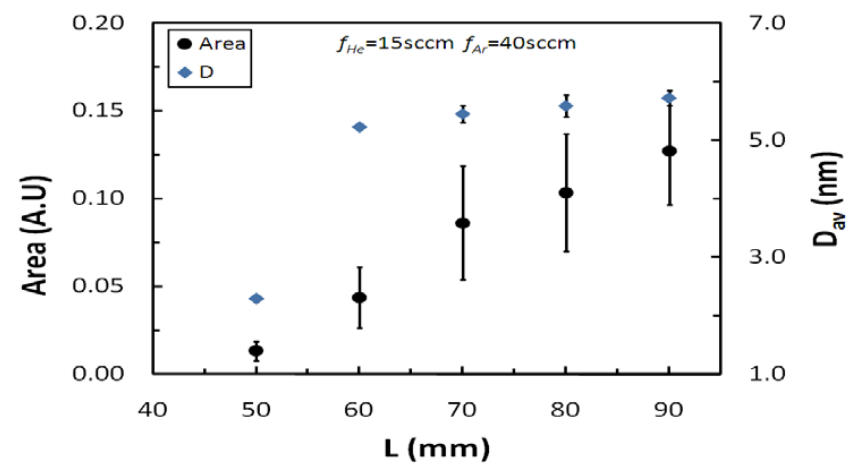

Figure 2: The dependence of the mean nanocluster diameter and area unde the size distribution curves on $\mathrm{f}_{\mathrm{He}}$

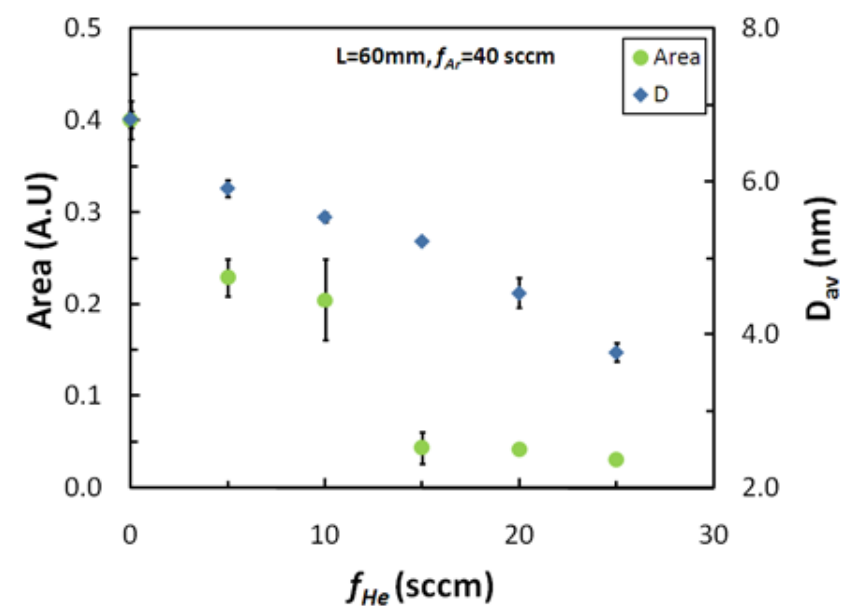

Figure 3: The dependence of the mean nanocluster diameter and area under the size distribution curves on $\mathrm{L}$. 
conduction is dominated by tunneling [19]. Therefore, it is assumed that the nanoclusters film is consisting of isolated groups of connected nanoclusters and the conduction happens along the main conduction paths, which contain several tunneling barriers. The I(V) temperature profile is converted into a conductance $\mathrm{G}(\mathrm{V})$ temperature profile using $\mathrm{G}=\mathrm{I} / \mathrm{V}$, as shown in Figure 5(a). The figure shows that the conductance increases with temperature. Furthermore, the slop of the conductance decreases as a function of temperature. The conductance was fitted to a conductance model $[20,21]$ :

$$
G(V)=\sigma_{0}(T) \exp \left[e V /\left(n k_{B} T\right)\right]
$$

where $\sigma_{0}(\mathrm{~T})$ is the temperature dependant conductance at $0 \mathrm{~V}$, e is the electron charge, $\mathrm{V}$ is the applied voltage across the nanocluster network, $\mathrm{k}_{\mathrm{B}}$ is Boltzmann constant, and $\mathrm{n}$ is the number of potential barriers between the nanoclusters forming the conduction path. The solid lines in Figure 5(a) are the linear fits of the G(V) profile according to Eq. 1. The figure reveals an acceptable agreement between the experimental results and the linear fit.

The y-intercepts $\left(\operatorname{Ln}\left(\sigma_{0}\right)\right)$ in Figure 5(a) are plotted as a function of $1 / \mathrm{T}$ in Figure 5(b). The figure reveals a linear relationship which confirms the Arrhenius behavior of the current nanocluster system. It is a typical behavior for isolated groups of connected nanoclusters. The slope of the curve was used to determine the activation energy $\left(\mathrm{E}_{\mathrm{a}}\right)$ associated with the transport into these islands to $9.3 \mathrm{meV}$. The nanocluster size $(6.7 \mathrm{~nm})$ was used to estimate the single-nanocluster Coulomb blockade energy $\left(E_{C}=e^{2} /(2 C)\right)$. Here $C$ was calculated assuming spherical nanoclusters $\left(C=4 \pi \varepsilon_{0} r\right.$, where $\varepsilon_{0}=8.85 \times 10$ ${ }^{12} \mathrm{C}^{2} / \mathrm{Nm}^{2}$, and $\mathrm{r}$ is the radius of the nanocluster). The calculation gives a Coulomb blockade of $214 \mathrm{meV}$ which is much greater than the activation energy. Since this estimation is much greater than the experimental activation energy, it suggests that the nanoclusters undergo fusing during the formation of nanocluster network.
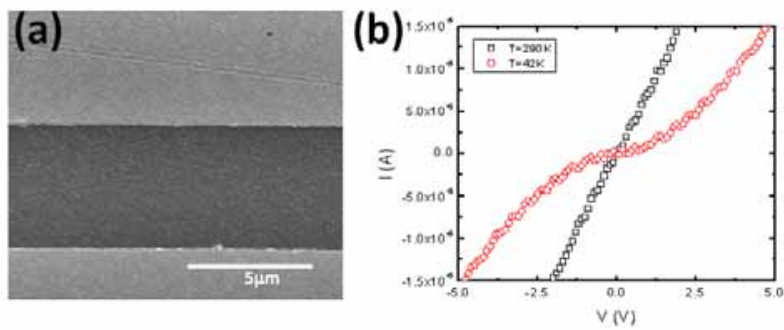

Figure 4: a) SEM image of the produced device between the two electrodes. b) I(V) curves of the device at 290 and $42 \mathrm{~K}$
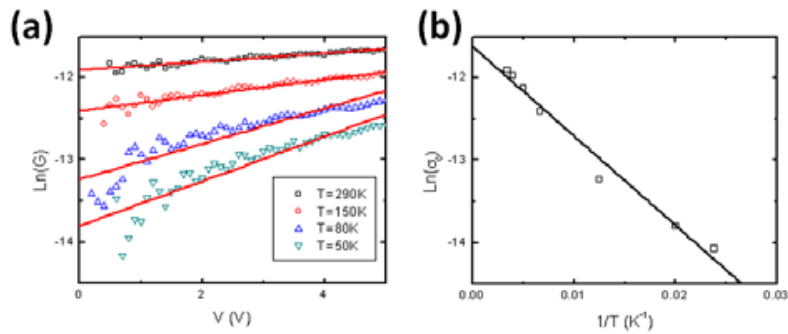

Figure 5: a) The dependence of $\operatorname{Ln}(G)$ on $V$ for the produced device. b) The dependence of $\operatorname{Ln}\left(\sigma_{0}\right)$ on temperature.

\section{Conclusion}

The effect of source conditions on the size and number of palladium (Pd) nanoclusters are investigated in this work. The results showed that as Helium flow rate increases, the mean nanocluster diameter decreases owing to high drift velocity of nanoclusters which reduces the nucleation time. Furthermore, the nanocluster size increases with the aggregation length due to a long nucleation time the nanoclusters spent in the aggregation region. For a constant Ar flow rate, the number of measured nanoclusters decreases with increasing Helium flow rate which could be attributed to the low sputtering yield of Helium gas. Thus, the probability of nanocluster formation via three-body collision mechanism decreases. This study demonstrates that using Helium inert gas can be used to minimize the average size and control the number of produced nanoclusters.

The electronic transport in palladium nanocluster devices are investigated via current-voltage $(\mathrm{I}(\mathrm{V}))$ measurements. The results show that the conductance through the device is dominated by charge carrier tunneling. The conduction temperature profile is fitted into a conduction model which enables the calculation of useful physical parameters. The conductance at $0 \mathrm{~V}$ is thermally activated with activation energy $9.3 \mathrm{meV}$ which is much less than the calculated Coulomb blockade energy. It suggests that some of the nanoclusters within the conduction path are fused together.

\section{Acknowledgments}

This work is funded by the Office of Research Support and Sponsored Projects (RSSP) at UAE University under a grant number 1592-02-2-01/10. The authors would like to thank Mr. S. Tariq at FMHS, United Arab Emirates University for the TEM imaging.

\section{References}

1. Watari N, Ohnishi S, Ishii Y (2000) Hydrogen storage in Pd clusters. J Phys Condens Matter 12: 6799.

2. Hanneken JW, Baker DB, Conradic MS, Eastmand JA (2002) NMR study of the nanocrystalline palladium-hydrogen system. J Alloys Compd 330-332 714-717.

3. Van Lith J, Lassesson A, Brown SA, Schulze M, Partridge JG, et al. (2007) A hydrogen sensor based on tunneling between palladium clusters. Appl Phys Lett 91: 181910.

4. Wsuurschum R, Reimann K, Grub S, Kubler A, Scharwaechter P, et al. (1997) Structure and diffusional properties of nanocrystalline Pd. Philos Mag B 76 : 407-417.

5. Kim SC, Shim WG, Ryu JY (2010) Effect of the growth of nano-sized Pd particle in $1 \mathrm{wt} \% \mathrm{Pd} / \mathrm{gamma}-\mathrm{Al} 2 \mathrm{O} 3$ catalyst on the complete oxidation of volatile organic compounds. J Nanosci Nanotechnol 10: 3521-3524.

6. Gibilisco S, Di Vece M, Palomba S, Faraci G, Palmer RE (2006) Pinning of size-selected Pd nanoclusters on graphite. J Chem Phys 125: 084704.

7. Brault P, Thomann AL, Andreazza-Vignolle C, Andreazza P (2002) Tuning growth from clusters to continuous ultrathin films: Experiments and molecular dynamics simulations of Pd plasma sputter deposition. Eur Phys J-Appl Phys 19: 83-87.

8. Pratontep S, Carroll SJ, Xirouchaki C, Streun M, Palmer RE (2005) Sizeselected cluster beam source based on radio frequency magnetron plasma sputtering and gas condensation. Rev Sci Instrum 76: 045103.

9. Wegner K, Walker B, Tsavros S, Pratsinis SE (2002) Design of metal nanoparticle synthesis by vapor flow condensation. Chem Eng Sci 57: 17531762.

10. Wahok K, Hihata T, Peng DL, Sumiyama K (1999) Compositional partition in $\mathrm{Ag}-\mathrm{Nb}$ alloy clusters produced by a plasma-gas-condensation cluster source. Nanostruct Mater 11: 1245-1251.

11. Simchi A, Ahmadi R, Reihani SM, Mahdavi A (2007) Kinetics and mechanisms of nanoparticle formation and growth in vapor phase condensation process. Materials and Design 28: 850-856. 
Citation: Ayesh Al, Mahmoud ST, Qamhieh N, Karam ZA (2012) Fabrication and Characterization of Percolating Nanocluster Devices. J Nanomed Nanotechol 3:143. doi:10.4172/2157-7439.1000143

Page 4 of 4

12. Ayesh Al, Qamhieh N, Ghamlouche H, Thaker S, EL-Shaer M (2010) Fabrication of size-selected Pd nanoclusters using a magnetron plasma sputtering source. J Appl Phys 107: 034317

13. Hihara T, Sumiyama K (1998) Formation and size control of a Ni cluster by plasma gas condensation. J App Phys 84: 5270.

14. Granqvist CG, Buhrman RA (1976) Ultrafine metal particles. J Appl Phys 47: 2200.

15. Xirouchaki C, Palmer RE (2004) Deposition of size-selected metal clusters generated by magnetron sputtering and gas condensation: a progress review. Phil Trans R Soc Lond A 362: 117-124.

16. Banerjee AN, Krishna R, Das B (2008) Size controlled deposition of Cu and Si nano-clusters by an ultra-high vacuum sputtering gas aggregation technique. Appl Phys A Mater Sci Process 90: 299-303.
17. Yamamuro S, Sumiyama K, Sakurai W, Suzuki K (1998) Cr cluster deposition by plasma-gas-condensation method. Supramolecular Science 5: 239-245.

18. Ayesh Al, Thaker S, Qamhieh N, Ghamlouche H (2011) Size-controlled Pd nanocluster grown by plasma gas-condensation method. J Nanopart Res 13 1125-1131.

19. Amman M, Wilkins R, Ben-Jacob E, Maker PD, Jaklevic RC (1991) Analytic solution for the current-voltage characteristic of two mesoscopic tunnel junctions coupled in series. Phys Rev B Condens Matter 43: 1146-1149.

20. Ozturk B, Blackledge C, Flanders BN, Grischkowsky D (2006) Reproducible interconnects assembled from gold nanorods. Appl Phys Lett 88: 073108.

21. Kaiser AB, Park YW (2005) Current-voltage characteristics of conducting polymers and carbon nanotubes. Mendeley 152: 181-184. 